\title{
Extreme-UV quiet Sun brightenings observed by the Solar Orbiter/EUI
}

\author{
D. Berghmans ${ }^{1}$, F. Auchère ${ }^{2}$, D. M. Long ${ }^{3}$, E. Soubriée, ${ }^{2,8}$, M. Mierla ${ }^{1,13}$, A. N. Zhukov ${ }^{1,9}$, U. Schühle ${ }^{4}$, P. Antolin ${ }^{10}$,
} L. Harra ${ }^{5,6}$, S. Parenti ${ }^{2}$, O. Podladchikova ${ }^{5}$, R. Aznar Cuadrado ${ }^{4}$, É. Buchlin ${ }^{2}$, L. Dolla $^{1}$, C. Verbeeck ${ }^{1}$, S. Gissot ${ }^{1}$, L. Teriaca ${ }^{4}$, M. Haberreiter ${ }^{5}$, A. C. Katsiyannis ${ }^{1}$, L. Rodriguez ${ }^{1}$, E. Kraaikamp ${ }^{1}$, P.J. Smith ${ }^{3}$, K. Stegen ${ }^{1}$, P. Rochus ${ }^{11}$, J. P. Halain ${ }^{11,12}$, L. Jacques ${ }^{11}$, W. T. Thompson ${ }^{7}$, and B. Inhester ${ }^{4}$

${ }^{1}$ Solar-Terrestrial Centre of Excellence - SIDC, Royal Observatory of Belgium, Ringlaan -3- Av. Circulaire, 1180 Brussels, Belgium e-mail: david.berghmans@oma.be

2 Université Paris-Saclay, CNRS, Institut d'Astrophysique Spatiale, 91405 Orsay, France

3 UCL-Mullard Space Science Laboratory, Holmbury St. Mary, Dorking, Surrey RH5 6NT, UK

${ }^{4}$ Max Planck Institute for Solar System Research, Justus-von-Liebig-Weg 3, 37077 Göttingen, Germany

5 Physikalisch-Meteorologisches Observatorium Davos, World Radiation Center, 7260 Davos Dorf, Switzerland

${ }^{6}$ ETH-Zürich, Wolfgang-Pauli-Str. 27, 8093 Zürich, Switzerland

7 Adnet Systems Inc., NASA Goddard Space Flight Center, Code 671, Greenbelt, MD 20771, USA

${ }^{8}$ Institute of Applied Computing \& Community Code, Universitat de les Illes Balears, 07122 Palma de Mallorca, Spain

9 Skobeltsyn Institute of Nuclear Physics, Moscow State University, 119992 Moscow, Russia

10 Department of Mathematics, Physics and Electrical Engineering, Northumbria University, Newcastle Upon Tyne NE1 8ST, UK

11 Centre Spatial de Liège, Université de Liège, Av. du Pré-Aily B29, 4031 Angleur, Belgium

12 European Space Agency (ESA/ESTEC), Keplerlaan 1, PO Box 299, 2200 AG Noordwijk, The Netherlands

13 Institute of Geodynamics of the Romanian Academy, Bucharest, Romania

Received 19 January 2021 / Accepted 1 April 2021

\section{ABSTRACT}

Context. The heating of the solar corona by small heating events requires an increasing number of such events at progressively smaller scales, with the bulk of the heating occurring at scales that are currently unresolved.

Aims. The goal of this work is to study the smallest brightening events observed in the extreme-UV quiet Sun.

Methods. We used commissioning data taken by the Extreme Ultraviolet Imager (EUI) on board the recently launched Solar Orbiter mission. On 30 May 2020, the EUI was situated at 0.556 AU from the Sun. Its High Resolution EUV telescope $\left(\mathrm{HRI}_{\mathrm{EUV}}, 17.4 \mathrm{~nm}\right.$ passband) reached an exceptionally high two-pixel spatial resolution of $400 \mathrm{~km}$. The size and duration of small-scale structures was determined by the $\mathrm{HRI}_{\mathrm{EUV}}$ data, while their height was estimated from triangulation with simultaneous images from the Atmospheric Imaging Assembly (AIA) on board the Solar Dynamics Observatory mission. This is the first stereoscopy of small-scale brightenings at high resolution.

Results. We observed small localised brightenings, also known as 'campfires', in a quiet Sun region with length scales between $400 \mathrm{~km}$ and $4000 \mathrm{~km}$ and durations between $10 \mathrm{~s}$ and $200 \mathrm{~s}$. The smallest and weakest of these HRI $\mathrm{I}_{\mathrm{EUV}}$ brightenings have not been previously observed. Simultaneous observations from the EUI High-resolution Lyman- $\alpha$ telescope $\left(\mathrm{HRI}_{\text {Lya }}\right.$ ) do not show localised brightening events, but the locations of the $\mathrm{HRI}_{\mathrm{EUV}}$ events clearly correspond to the chromospheric network. Comparisons with simultaneous AIA images shows that most events can also be identified in the $17.1 \mathrm{~nm}, 19.3 \mathrm{~nm}, 21.1 \mathrm{~nm}$, and $30.4 \mathrm{~nm}$ pass-bands of AIA, although they appear weaker and blurred. Our differential emission measure analysis indicated coronal temperatures peaking at $\log T \approx 6.1-6.15$. We determined the height for a few of these campfires to be between 1000 and $5000 \mathrm{~km}$ above the photosphere. Conclusions. We find that 'campfires' are mostly coronal in nature and rooted in the magnetic flux concentrations of the chromospheric network. We interpret these events as a new extension to the flare-microflare-nanoflare family. Given their low height, the EUI 'campfires' could stand as a new element of the fine structure of the transition region-low corona, that is, as apexes of small-scale loops that undergo internal heating all the way up to coronal temperatures.

Key words. Sun: UV radiation - Sun: transition region - Sun: corona - instrumentation: high angular resolution

\section{Introduction}

The Extreme Ultraviolet Imager (EUI, Rochus et al. 2020) on board the Solar Orbiter (Müller et al. 2020) consists of three telescopes: the Full Sun Imager (FSI), which operates in two extreme UV bandpasses $(17.4 \mathrm{~nm}$, and $30.4 \mathrm{~nm})$, and two High Resolution Imagers (HRIs).
The telescope labelled 'HRI EUV' has a bandpass centred at $17.4 \mathrm{~nm}$ and is dominated by Fe IX and Fe X emission at $1 \mathrm{MK}$. The telescope labelled ' $\mathrm{HRI}_{\mathrm{Lya}}$ ' has a bandpass centred at $121.6 \mathrm{~nm}$ and is dominated by the Lyman- $\alpha$ line of hydrogen. The scientific goal of the two HRIs is to observe the fine structure and dynamics, respectively, in the upper chromosphere $\left(\mathrm{HRI}_{\mathrm{Lya}}\right)$ 
and in the low corona ( $\left.\mathrm{HRI}_{\mathrm{EUV}}\right)$. The transition region between these two layers is where the temperature abruptly increases by two orders of magnitude and where the replenishment of energy and mass that sustains the higher corona and, hence, the whole heliosphere, takes place. Its physics is very complex and it is clear that the crucial processes responsible for the formation of the corona must be operating at very small spatial and temporal scales at all times (Parker 1988; Klimchuk 2015).

The classical picture of the transition region is that of a thermal interface between the coronal and chromospheric plasma (Gabriel 1976). However, it cannot fully describe the high radiance of the transition region lines, so the concept of the "unresolved fine structure" (UFS) of the transition region in the quiet Sun has been proposed (see Feldman 1983; Dowdy et al. 1986): a plethora of small-scale low-lying loops that are magnetically isolated from the corona. Such structures were later detected with the improvement of the spatial resolution of the observations (e.g. Feldman et al. 1999; Warren \& Winebarger 2000). More recently, Hansteen et al. (2014) used the data from the Interface Region Imaging Spectrograph (IRIS, De Pontieu et al. 2014 , with a spatial resolution of $0.4^{\prime \prime}, 290 \mathrm{~km}$ ), and found that low-lying loops (projected full lengths of 4-12 Mm) at low transition-region temperatures ( $\log T$ around 4.3-4.8) to be a major constituent of the UFS. The High Resolution Coronal Imager (Hi-C) sounding rocket (Kobayashi et al. 2014; Rachmeler et al. 2019) reached the highest resolution in extreme UV coronal observations so far $\left(0.3^{\prime \prime}, 218 \mathrm{~km}\right)$ and detected fine scale features of less than $200 \mathrm{~km}$ wide (Peter et al. 2013; Barczynski et al. 2017). However, thus far, Hi-C has only observed active regions, so the fine structure of the upper transition region of the quiet Sun remains unexplored.

Earlier studies of the quiet Sun revealed a wide range of extreme UV brightenings at transition region and low coronal temperatures, often interpreted in terms of nanoflare heating. We use the term 'nanoflare' to refer to the energy range, regardless of the driving mechanism. For coronal heating purposes, an increasing number of miniature heating events are required at scales that are progressively smaller in length, characterised by a power law distribution with an index steeper than -2 (Hudson 1991; Berghmans 2002; Joulin et al. 2016). A power-law distribution was found for the number of events as a function of their energy content (Benz \& Krucker 2002; Aschwanden 2004). A powerlaw index close to -2 was found by Berghmans et al. (1998), whereas a power-law index of -2.5 was found by Harra et al. (2000). However, Aschwanden \& Parnell (2002) found a power law of index -1.54 , which matches the distribution of active region transient brightenings (Shimizu et al. 1994) and hard Xray flares (Crosby et al. 1993). The authors explained the difference in terms of a temperature bias imposed by narrow band extreme UV imagers. Recently, Chitta et al. (2021) analysed 'EUV bursts' and reached the conclusion that at the resolution of the images of the AIA instrument on board the Solar Dynamics Observatory (SDO, Pesnell et al. 2012), there was, by a factor of 100 , an insufficient number of events to account for the energy that is required to heat the corona. At transition region temperatures, explosive events are seen in spectroscopic data with lifetimes of order of minutes and sizes below $1 \mathrm{~mm}$ (Brueckner \& Bartoe 1983; Dere et al. 1989; Teriaca et al. 2004).

This work uses the first release of properly calibrated and formatted EUI data in December 2020 ${ }^{1}$. Before that date, only uncalibrated, quick-look EUI data were publicly available for outreach events, such as the press release of the European Space

https://doi.org/10.24414/wvj6-nm32
Agency in July 2020 featuring the EUI team's first presentation of 'campfires'. The objective of this Letter is to present novel observations of the EUI/HRIs (Sect. 2) and to discuss what they may contribute to our understanding of coronal structuring and heating (Sect. 3).

\section{Observations}

As part of the instrument commissioning, the two EUI HRI telescopes were operated on 30 May 2020, for the first time in parallel, at a fast imaging cadence of $5 \mathrm{~s}$ and acquired 50 images each. Solar Orbiter was located at $31.5^{\circ}$ west in solar longitude from the Earth-Sun line (see Fig. 1). In this study, we also use the data acquired by the AIA instrument (Lemen et al. 2012) on board the SDO (Pesnell et al. 2012) in Earth orbit. To compare EUI and AIA across the whole field of view (FOV), we remapped the images from both instruments (see Appendix A) to a regular $2400 \times 2400$ grid in Carrington coordinates with a 0.01625 heliographic degrees $(197 \mathrm{~km})$ pitch, which corresponds to the original HRI $\mathrm{EUV}_{\mathrm{EU}}$ resolution. Appendix A contains the details of the acquired data set.

In Fig. 1, we show the HRI $I_{\text {EUV }}$ field of view. The subject of the present study are the small-scale brightenings appearing in $\mathrm{HRI}_{\mathrm{EUV}}$ images with sizes down to the two-pixel resolution limit of the telescope. These brightenings have become known as 'campfires', a term which we use for 'small brightenings observed by $\mathrm{HRI}_{\mathrm{EUV}}$ ' without necessarily implying that these are physically different from previously observed small extreme UV brightenings in the corona.

We used an automated detection method to determine the statistical significance of small features spatially and track them in time through the sequence. The detection scheme (see Appendix B) applied to the stack of 50 Carrington projected HRI $_{\text {EUV }}$ images revealed 1467 campfires. Dividing this number by the area of the field of view and by the duration of our sequence, we arrive at a birthrate of $3.7 \times 10^{-17} \mathrm{~m}^{-2} \mathrm{~s}^{-1}$, which is an order of magnitude lower than the birthrate of explosive events (Teriaca et al. 2004 found $4.1 \times 10^{-16} \mathrm{~m}^{-2} \mathrm{~s}^{-1}$ ).

Figure 2 shows the histograms of the projected area, duration, and total intensity of these events. We note that we did not remove the left-most bins of the histograms in Fig. 2 that correspond to events that appear only in one image or only in one pixel. Since these bins are in agreement with the trend seen at larger scales, we kept them in the figure as an illustration that the campfires population extends consistently down to the limitations of our data set in terms of spatial and temporal resolution. Individual events of one pixel in a single frame cannot be confirmed, but some one-pixel events can last several frames and some one-frame events consist of several pixels. Out of the 1467 events, there are 558 which have both their projected area larger than one pixel and their duration larger than five sec (the imaging cadence).

The detected population is limited by the total duration of our HRI EUV data set, which is only $245 \mathrm{~s}$. The longest event durations in our data set approaches $200 \mathrm{~s}$, but among these, many events are truncated at the end or the start of the sequence. There is no such artificial truncation in the histogram of the projected area, where the largest events are on the order of a few $\mathrm{Mm}^{2}$, which corresponds to roughly one hundred pixels. Strikingly, both the histogram of projected area and the histogram of campfire duration show a power law-like behaviour stretching across at least two orders of magnitude.

Many events have an elongated nature, reminiscent of small loop-like structure. Linear (projected) lengths of these elongated 


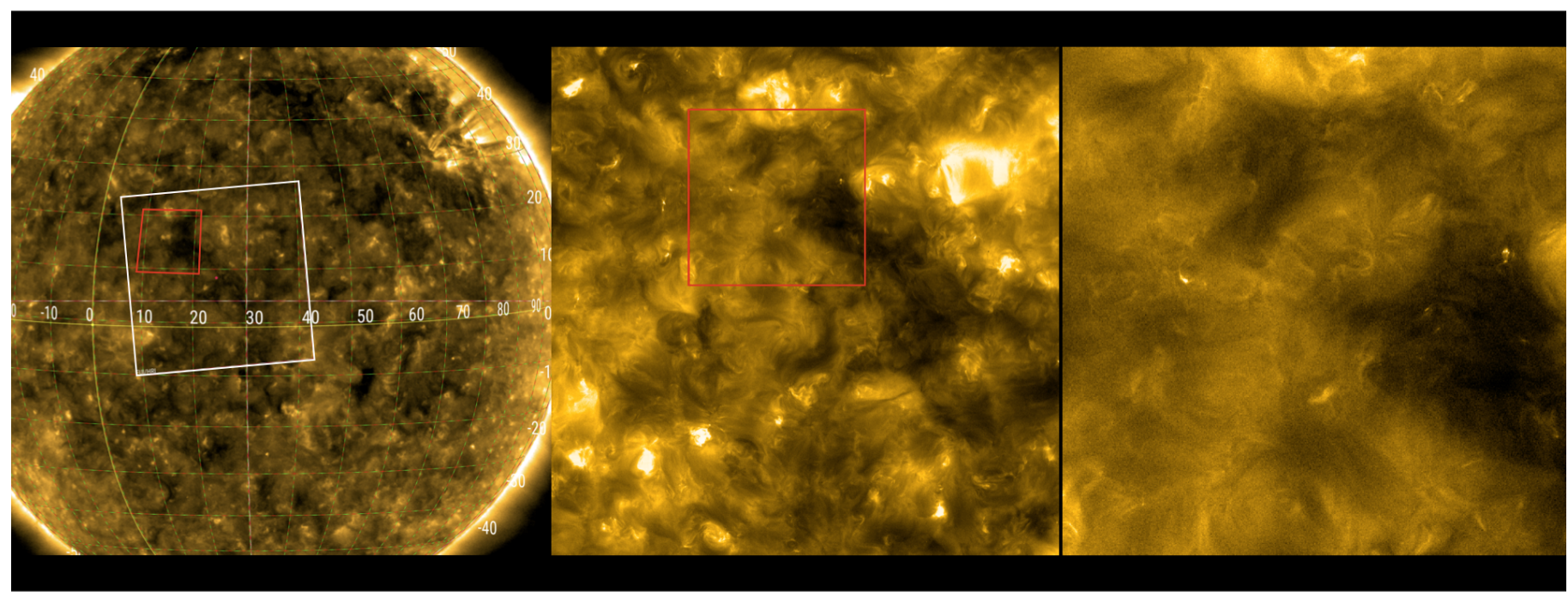

Fig. 1. Overview of the solar corona of 30 May 2020 as seen by EUI on board Solar Orbiter. Left: location of the HRI field of view is shown as a white square on an EUI/FSI $17.4 \mathrm{~nm}$ image. Stonyhurst coordinates are shown, with the crossing of the two yellow great circles indicating the Earth/SDO sub-solar point. We note that the spacecraft roll angle is aligned with the 'north' direction of the orbital plane of the Solar Orbiter - and not with the solar meridional plane. The image visualisation is from JHelioviewer (Müller et al. 2017). Middle: first image of the HRI $\mathrm{EUV}_{\mathrm{E}}$ sequence. Right: sub-field corresponding to the red square indicated in the left two panels. The brightest features correspond to the "campfires" studied in this paper.
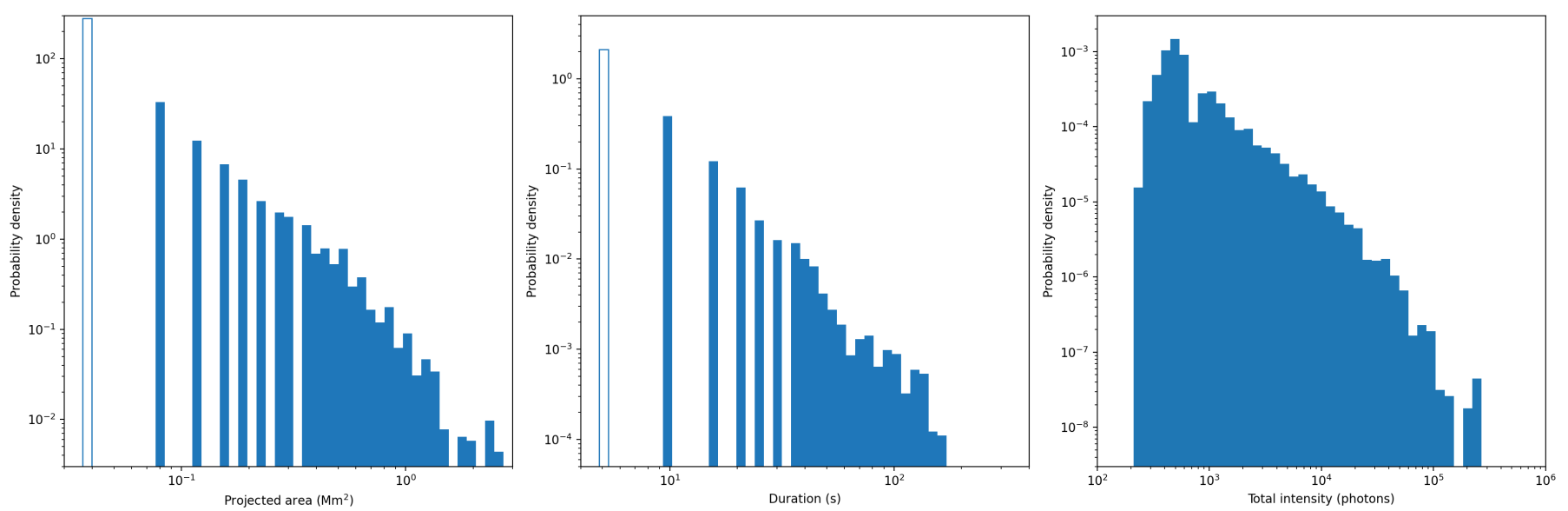

Fig. 2. Observed event distribution as a function of projected area, duration, and total intensity. Projected area refers to all the pixel area that the events occupy at some stage over their duration. The probability density is scaled such that when integrated over all bin widths, the total event count of 1467 is obtained. The smallest areas and durations (only one pixel or one exposure) are displayed as bars without filling.

structures range from 1 to 20 pixels $(0.2 \mathrm{~mm}$ to $4 \mathrm{~mm})$ with aspect ratios distributed mostly between 1 and 5. The smallest observed structures are similar to the smallest active region loops observed by Hi-C (Peter et al. 2013).

As expected from the histograms in Fig. 2, most campfires are very close to the $\mathrm{HRI}_{\mathrm{EUV}}$ resolution limit and are therefore either dot-like (Fig. 3) or short loop-like (Fig. 4). In most cases, these small events can be recognised in the SDO/AIA bandpasses at $21.1 \mathrm{~nm}, 19.3 \mathrm{~nm}, 17.1 \mathrm{~nm}$, and $30.4 \mathrm{~nm}$, although they typically appear weaker and fuzzier, which can be expected from the difference in spatial resolution. No clear event signatures were identified in any of the other SDO/AIA bandpasses. In Appendix $\mathrm{C}$, we show a few more examples, including bigger events with internal structure, including possibly interacting loops.

Remarkably, the observed campfires do not unambiguously reveal localised brightenings in $\mathrm{HRI}_{\text {Lya }}$ at the same location and time as the campfires detected in $\mathrm{HRI}_{\mathrm{EUV}}$. It is possible that the
$\mathrm{HRI}_{\text {Lya }}$ signature is a more fuzzy, distributed brightening of a bigger neighbourhood. This will be studied in a future work. In any case, the HRI $\mathrm{EUV}_{\mathrm{V}}$ campfires have a clear tendency to outline the chromospheric network as shown by $\mathrm{HRI}_{\text {Lya }}$. In the top panel of Fig. 5, we show the location of the campfires detected in the HRI $_{\text {EUV }}$ data, over-plotted as yellow dots on a reference $\mathrm{HRI}_{\text {Lya }}$ image, which was obtained by averaging over the 50 Carrington projected $\mathrm{HRI}_{\text {Lya }}$ images.

The intensity distribution of the averaged HRI $\mathrm{Lya}_{\text {a }}$ map is shown in the bottom panel of Fig. 5 (solid histogram). The number of detection events falling within each of the 50 equal intensity bins is displayed with crosses. The vertical dashed lines are arbitrarily chosen intensity levels encompassing the peak of the event distribution (crosses) and are used as contours on the intensity map (top panel). The brown and blue colours correspond in both panels to the same intensity levels. The detected events are generally not in the darker cell-centres and there are also very few of them at the brightest locations. The 


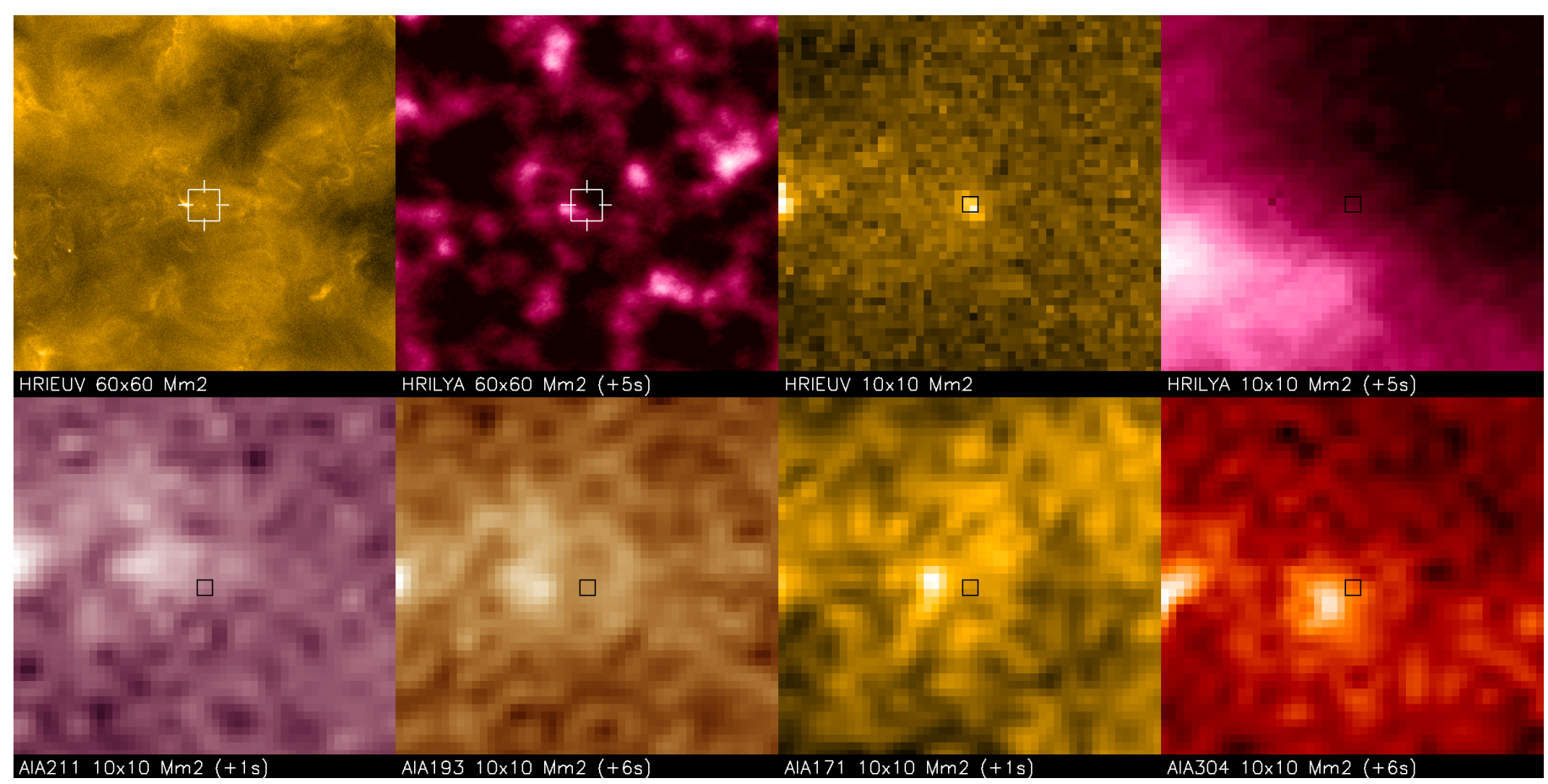

Fig. 3. Example of a dot-like event. The white square in the two top-left panels corresponds to the field of view of the other, zoomed-in panels.

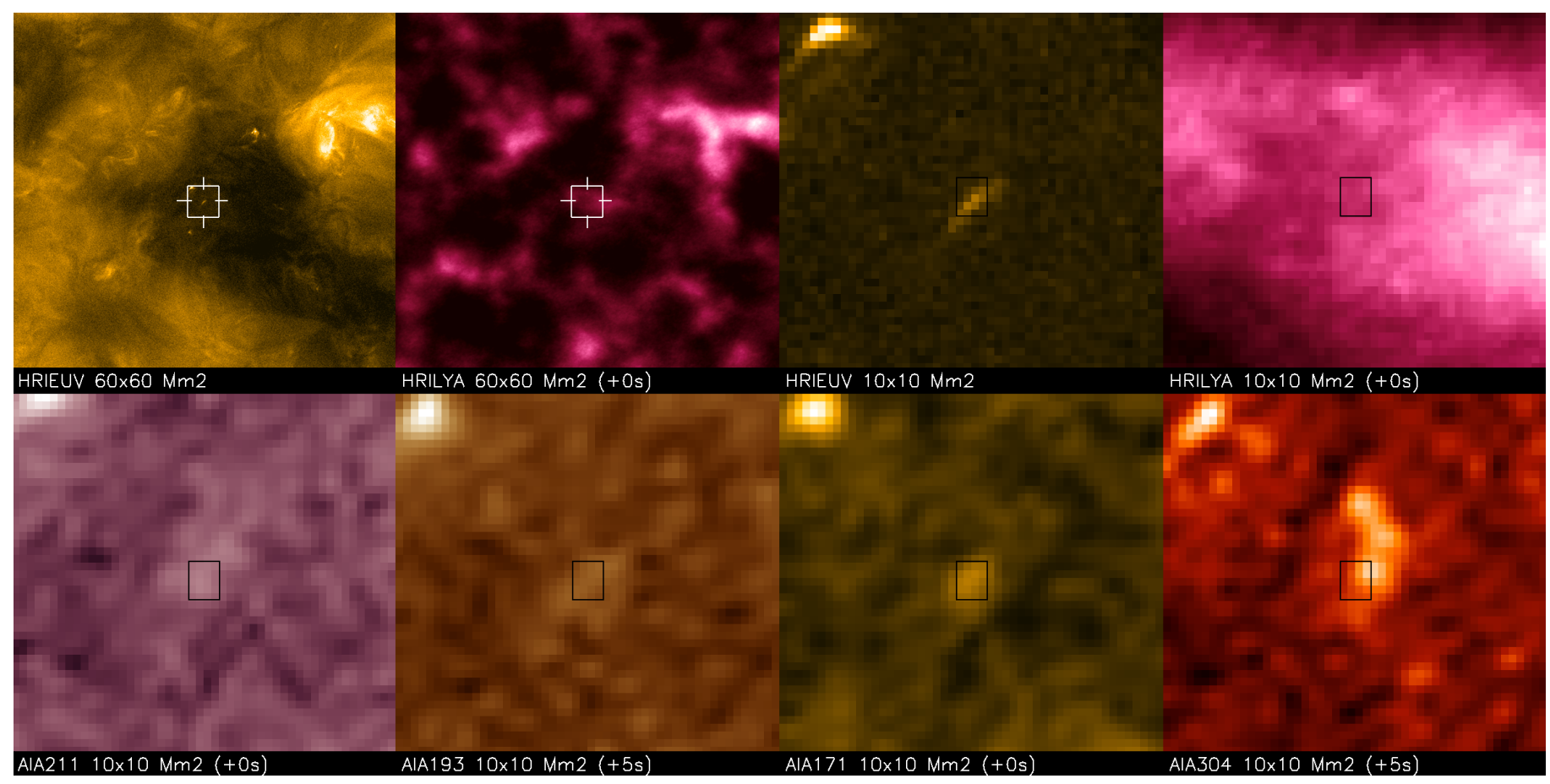

Fig. 4. Example of a loop-like event. The white square in the two top-left panels corresponds to the field of view of the other, zoomed-in panels.

majority of the campfires are located at the network boundaries, which is the same location as where explosive events are found (Porter \& Dere 1991). We note that in contrast to the SDO/AIA images, the $\mathrm{HRI}_{\text {Lya }}$ images are taken from exactly the same perspective as $\mathrm{HRI}_{\mathrm{EUV}}$. Projection effects are therefore not likely to have a major influence on the apparent location of $\mathrm{HRI}_{\mathrm{EUV}}$ events in the HRI Lya images.

In order to determine the height of campfires above the photospheric surface, we performed triangulation on sixteen small campfires that could be identified on both $\mathrm{HRI}_{\mathrm{EUV}} 17.4 \mathrm{~nm}$ and
AIA $17.1 \mathrm{~nm}$ images. Figure 6 shows the height above the photosphere versus the projected length for 16 campfires selected for an easy identification. The projected length of each campfire is calculated as the distance between the farthest two pixels of the campfire in the image plane. The photospheric radius value of $695700 \mathrm{~km}$ (Prša et al. 2016) is subtracted to obtain the height above the photosphere. The error bars are derived in Appendix D. From Fig. 6, we can see that the campfires are located between $1000 \mathrm{~km}$ and $5000 \mathrm{~km}$ above the photospheric surface. 

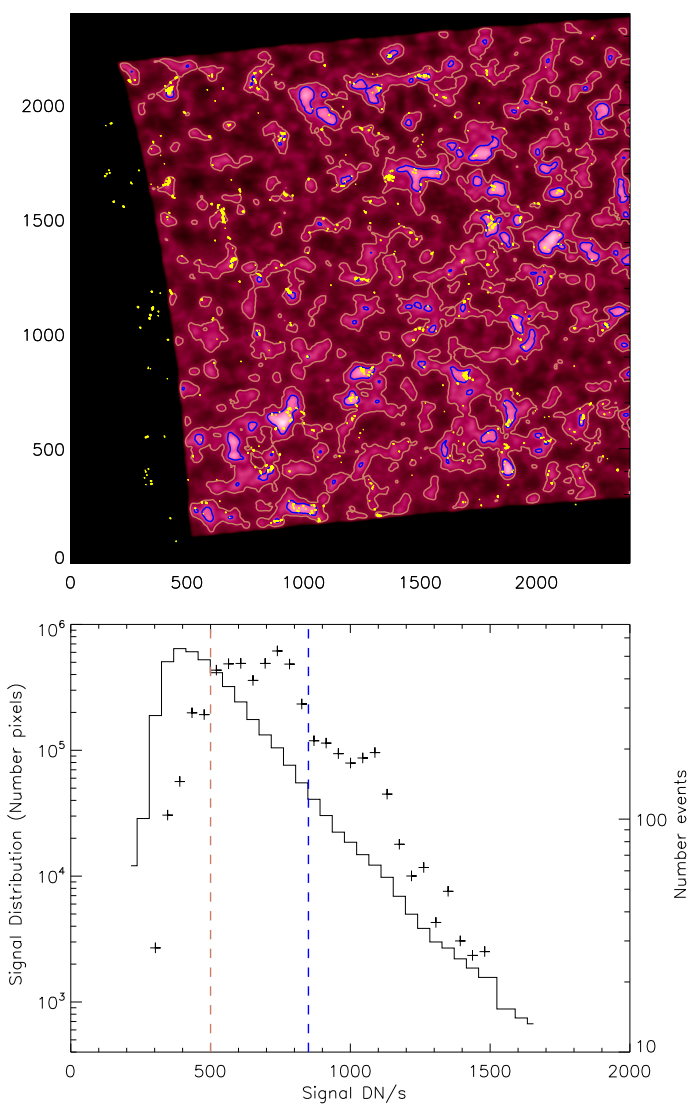

Fig. 5. Location of the HRI $\mathrm{EUV}_{\mathrm{EU}}$ campfires in the HRI $\mathrm{L}_{\mathrm{Lya}}$ images. Top: averaged intensity map of $50 \mathrm{HRI}_{\text {Lya }}$ images. Detected 'campfire' events are displayed in yellow. The contours correspond to signals of 500 $\mathrm{DN} / \mathrm{s}$ (brown) and $850 \mathrm{DN} / \mathrm{s}$ (blue). Bottom: intensity distribution of the $\mathrm{HRI}_{\text {Lya }}$ averaged map (solid histogram). The number of detected 'campfire' events present in each intensity bin is represented with crosses. Vertical dashed lines embed signals between $500 \mathrm{DN} / \mathrm{s}$ (brown) and 850 $\mathrm{DN} / \mathrm{s}$ (blue), where the majority of the detected events are present. Both $y$-axes are displayed in logarithmic scale.

The 3D geometry of campfires is unclear, as many of them are barely resolved. Various complex geometries could be envisaged, but here we start with the simplest assumption, namely, that campfires are semi-circular loops. A semi-circular loop has an apex at height $H$ which corresponds to half the length between the loop foot points $L$. The black $H=L / 2$ line in Fig. 6 is, by the definition of the apex, the upper limit for the height of any point on a semi-circular loop. For our 16 campfires, we triangulated the brightest pixel or the mid-point of a few bright pixels if there are several adjacent pixels of comparable brightness. Figure 6 shows that the resulting campfire heights are systematically higher than the $H=L / 2$ line. Figure 7 shows possible interpretations. A semi-circular loop (a) clearly does not explain the height observations. If campfires represent small-scale loops, then they are either (b) elongated (tall) loops or (c) only their apexes are visible. The latter possibility seems more likely as we do not expect the $17.4 \mathrm{~nm}$ emission to extend all the way down to the photosphere. Such a scenario is conceivable when, for instance, the observed campfire corresponds to emission near a contact point for nearby loops (d).

The temperatures of the events were estimated through differential emission measure (DEM) analysis, using the methods of Hannah \& Kontar (2012) and Cheung et al. (2015). Here, we show only the result obtained by the former method. The temper-

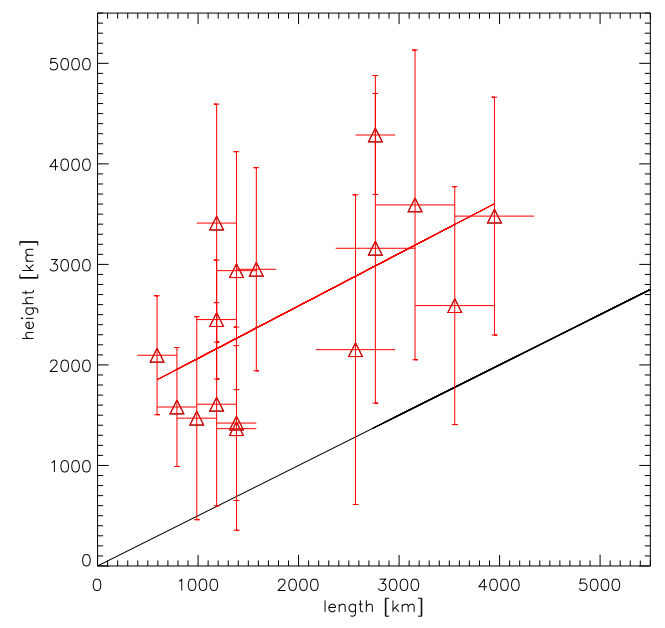

Fig. 6. Height above the photosphere of the 16 campfires versus their projected length (red triangles with their error bars, $h \pm \delta h$ ). The red line is a linear fit to the data points. The black line represents the height as half of the projected length as one would expect for a semi-circular loop, for comparison.

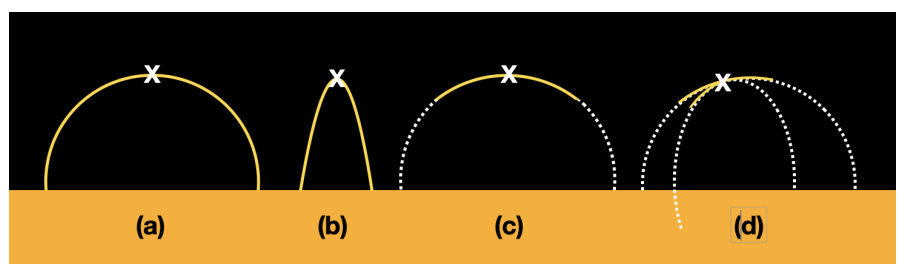

Fig. 7. Scenarios to interpret the campfire heights shown in Fig. 6. The yellow solid lines are the observed campfires, the dotted lines are the invisible loop segments, and the white cross is the triangulation point.

atures inferred this way are essentially the same as for the other method. In Fig. 8, we plot the 2D histogram of the total emission measure (EM) for each pixel (integrated over temperature) and the DEM-weighted temperature, as defined in Parenti et al. (2017). We exclude campfires with only one pixel or lasting only one frame. We also correct for the shift in location caused by the different viewpoints by interpolating each event to the location of its intensity barycentre. The temperature distribution peaks at $\log T \approx 6.1$, with a full width half maximum (FWHM) of $5.43(\log \mathrm{K})$ and with a relatively long tail extending to $2 \mathrm{MK}$. While this strongly indicates that campfires are coronal events, namely, that the plasma in these brightenings reaches temperatures of $1 \mathrm{MK}$, it should be noted that campfires may not always dominate the EM along the line of sight (LOS). Possibly, the DEM-weighted temperature is not always a good representative of the brightening temperature. Nonetheless, we note that both the emission measure and the temperature distribution of the events (but in particular the former) are clearly shifted towards higher values with respect to the background.

\section{Summary and discussion}

In a first high-cadence data set from the high-resolution telescopes of EUI, we identified a large number of extreme UV brightenings in the quiet Sun that are also referred to as 'campfires'. To our knowledge, this is the highest resolution image sequence of the quiet Sun corona ever achieved. Given the favourable $31.5^{\circ}$ separation in heliocentric longitude with SDO/AIA in Earth orbit, we were also able to apply triangulation 


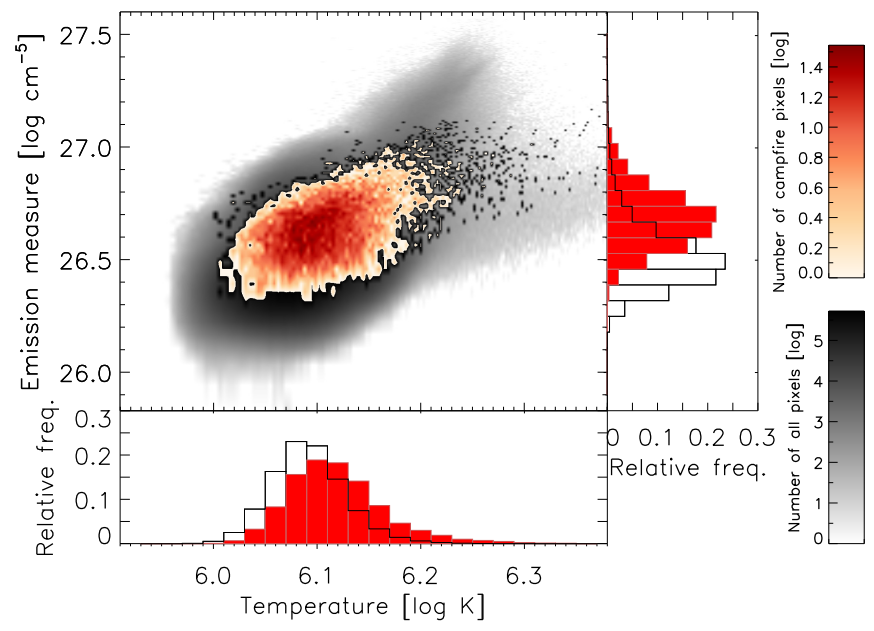

Fig. 8. 2D histogram of the total emission measure and DEM-weighted temperature for campfire pixels (red) and all pixels (black). The colour is given according to the logarithm of the number of pixels. The number of campfire pixels and all pixels are roughly 9800 and $1.1 \times 10^{8}$, respectively.

techniques at the highest resolution ever to determine the height of campfires. The characteristics of these campfires are summarised below.

An increasing number of campfires are found at progressively smaller scales, down to the resolution of the present HRI $_{\text {EUV }}$ data set. We find an apparent power law distributions for the campfire areas, durations, and total intensity. The area distribution is limited at the lower end by spatial resolution (a 2-pixel area is $0.08 \mathrm{~mm}^{2}$ ). At the higher end, the area distribution ends around $5 \mathrm{~mm}^{2}$, which is a consequence of our wavelet based detection scheme. The duration distribution is limited at the lower end by the temporal resolution of the present data set (twice the image cadence is $10 \mathrm{~s}$ ). At the higher end, the duration distribution ends around $200 \mathrm{~s}$, which is close to the total duration of our data set, namely $245 \mathrm{~s}$. The appearance of the campfires at progressively smaller scales ranges from small systems of possible interacting loops to individual loops and, finally, to dots at the resolution limit of the data set. The aspect ratios of length versus width range from 1 to 5 .

The observed campfires observed in $\mathrm{HRI}_{\mathrm{EUV}}$ do not, obviously, have corresponding localised brightenings in $\mathrm{HRI}_{\mathrm{Lya}}$ at the same time and place. However, the events detected in HRI $\mathrm{HUV}_{\mathrm{EU}}$ do line up along the chromospheric network as observed by $\mathrm{HRI}_{\text {Lya }}$. A comparison with simultaneous AIA images shows that most events can be found back in $21.1 \mathrm{~nm}, 19.3 \mathrm{~nm}, 17.1 \mathrm{~nm}$, and $30.4 \mathrm{~nm}$ as well, however, as compared to HRI $\mathrm{EUV}_{\text {, they }}$ appear weaker and fuzzier, which is as expected given the difference in resolution. In addition, they are not seen in 'hotter' AIA bandpasses.

Using triangulation techniques, we determined for a subsample of events that the campfires are located between $1000 \mathrm{~km}$ and $5000 \mathrm{~km}$ above the photosphere. Campfires are events at coronal temperatures: the distribution of their DEM-weighted temperatures peaks at $\log T \approx 6.12$, with a FWHM of 0.074 $(\log \mathrm{K})$.

Using Hi-C data, Tiwari et al. (2019) reported energy-release events that resemble some of the bigger campfires observed by $\mathrm{HRI}_{\mathrm{EUV}}$, but they only analysed active region observations. In the quiet Sun, the smallest extreme UV brightenings have been observed with SDO/AIA (Joulin et al. 2016; Chitta et al. 2021). The superior spatial and temporal resolution of the studied $\mathrm{HRI}_{\text {EUV }}$ quiet Sun data set allows us to extend the family of extreme UV quiet Sun brightenings down to spatial scales less than half a Mm and down to temporal scales of $10 \mathrm{~s}$.

Campfires are located above the chromospheric network, where the strongest quiet Sun magnetic fields reside. This suggests that campfires are magnetically defined features, as is further supported by their often isolated, distinct loop-like appearance. While the campfires do reach coronal temperatures (1-1.6 MK), they are located at remarkably low heights, between $1000 \mathrm{~km}$ and $5000 \mathrm{~km}$ from the photosphere. We believe it is the first time such low heights of quiet sun structures emitting around $17.4 \mathrm{~nm}$ have been determined. Compared to their lengths, the campfires are systematically higher than what could be expected for semi-circular loops. It is either the case that campfires are vertically elongated loops or that campfires correspond only to loop tops that are brightening. We propose that campfires are the apexes of low-lying small-scale network loops that are heated to coronal temperatures.

The observed heights of campfires at coronal temperatures coincide with the heights of the cold transition region loops reported by Hansteen et al. (2014). This co-existence is coherent with the picture of a plethora of small-scale low lying loops existing at different temperatures (Feldman 1983; Dowdy et al. 1986). Future research will address the reasons for which the campfire loops stand out as particularly hot when compared to neighbouring loops that do not surpass transition region temperatures.

The bigger campfires have durations and sizes that are comparable to explosive events (Dere et al. 1989), even if the latter ones are rather observed at transition region temperatures; see also Peter et al. (2014). In addition, they both appear at network boundaries. Campfires are possibly an order of magnitude less frequent than explosive events, although this might be a detection bias as explosive events are spectroscopically identified. Some of the AIA $17.1 \mathrm{~nm}$ quiet Sun small brightenings studied by Innes \& Teriaca (2013) are clearly associated with spectral profiles that are typical of explosive events. Due to the lack of spectroscopic data, this aspect, unfortunately, could not be checked for the campfire population.

The current observations were taken at a distance of $0.556 \mathrm{AU}$ from the Sun. Future perihelia of Solar Orbiter will go down to below $0.3 \mathrm{AU}$ and increase the effective spatial resolution of $\mathrm{HRI}_{\mathrm{EUV}}$ by another factor of 2 . Joint campaigns with other remote sensing instruments on board the Solar Orbiter, such as the Polarimetric and Helioseismic Imager (PHI, Solanki et al. 2020) and the SPICE spectrograph (Spice Consortium 2020), will help to confirm or discard our interpretation. This is particularly important for determining the driver of these heating events, their link to explosive events, and their role in coronal heating.

Acknowledgements. The building of EUI was the work of more than 150 people during more than 10 years, we gratefully acknowledge all the efforts that have led to a successfully operating instrument. The authors thank the Belgian Federal Science Policy Office (BELSPO) for the provision of financial support in the framework of the PRODEX Programme of the European Space Agency (ESA) under contract number 4000112292 . The French contribution to the EUI instrument was funded by the French Centre National d'Etudes Spatiales (CNES); the UK Space Agency (UKSA); the Deutsche Zentrum für Luft- und Raumfahrt e.V. (DLR); and the Swiss Space Office (SSO). PA and DML acknowledge funding from STFC Ernest Rutherford Fellowships No. ST/R004285/2 and ST/R003246/1, respectively. SP acknowledges the funding by CNES through the MEDOC data and operations center. 
D. Berghmans et al.: Extreme-UV quiet Sun brightenings observed by the Solar Orbiter/EUI

\section{References}

Aschwanden, M. J. 2004, Physics of the Solar Corona An Introduction (Praxis Publishing Ltd.)

Aschwanden, M. J., \& Parnell, C. E. 2002, ApJ, 572, 1048

Barczynski, K., Peter, H., \& Savage, S. L. 2017, A\&A, 599, A137

Benz, A. O., \& Krucker, S. 2002, ApJ, 568, 413

Berghmans, D. 2002, in Solar Variability: From Core to Outer Frontiers, ed. A.

Wilson, ESA Spec. Publ., 2, 501

Berghmans, D., Clette, F., \& Moses, D. 1998, A\&A, 336, 1039

Brueckner, G. E., \& Bartoe, J. D. F. 1983, ApJ, 272, 329

Cheung, M. C. M., Boerner, P., Schrijver, C. J., et al. 2015, ApJ, 807, 143

Chitta, L. P., Peter, H., \& Young, P. R. 2021, A\&A, 647, A159

Crosby, N. B., Aschwanden, M. J., \& Dennis, B. R. 1993, Sol. Phys., 143, 275

De Pontieu, B., Title, A. M., Lemen, J. R., et al. 2014, Sol. Phys., 289, 2733

Dere, K. P., Bartoe, J. D. F., \& Brueckner, G. E. 1989, Sol. Phys., 123, 41

Dowdy, J. F., Rabin, D., \& Moore, R. L. 1986, Sol. Phys., 105, 35

Feldman, U. 1983, ApJ, 275, 367

Feldman, U., Widing, K. G., \& Warren, H. P. 1999, ApJ, 522, 1133

Gabriel, A. H. 1976, Philos. Trans. R. Soc. London Ser. A, 281, 339

Hannah, I. G., \& Kontar, E. P. 2012, A\&A, 539, A146

Hansteen, V., De Pontieu, B., Carlsson, M., et al. 2014, Science, 346, 1255757

Harra, L. K., Gallagher, P. T., \& Phillips, K. J. H. 2000, A\&A, 362, 371

Hudson, H. S. 1991, Sol. Phys., 133, 357

Inhester, B. 2006, ArXiv e-prints [arXiv: astro-ph/0612649]

Innes, D. E., \& Teriaca, L. 2013, Sol. Phys., 282, 453

Joulin, V., Buchlin, E., Solomon, J., \& Guennou, C. 2016, A\&A, 591, A148
Klimchuk, J. A. 2015, Philos. Trans. R. Soc. London Ser. A, 373, 20140256

Kobayashi, K., Cirtain, J., Winebarger, A. R., et al. 2014, Sol. Phys., 289, 4393

Lemen, J. R., Title, A. M., Akin, D. J., et al. 2012, Sol. Phys., 275, 17

Müller, D., Nicula, B., Felix, S., et al. 2017, A\&A, 606, A10

Müller, D., St. Cyr, O. C., Zouganelis, I., et al. 2020, A\&A, 642, A1

Murtagh, F., Starck, J. L., \& Bijaoui, A. 1995, A\&AS, 112, 179

Parenti, S., del Zanna, G., Petralia, A., et al. 2017, ApJ, 846, 25

Parker, E. N. 1988, ApJ, 330, 474

Pesnell, W. D., Thompson, B. J., \& Chamberlin, P. C. 2012, Sol. Phys., 275, 3

Peter, H., Bingert, S., Klimchuk, J. A., et al. 2013, A\&A, 556, A104

Peter, H., Tian, H., Curdt, W., et al. 2014, Science, 346, 1255726

Porter, J. G., \& Dere, K. P. 1991, ApJ, 370, 775

Prša, A., Harmanec, P., Torres, G., et al. 2016, AJ, 152, 41

Rachmeler, L. A., Winebarger, A. R., Savage, S. L., et al. 2019, Sol. Phys., 294, 174

Rochus, P., Auchère, F., Berghmans, D., et al. 2020, A\&A, 642, A8

Shimizu, T., Tsuneta, S., Acton, L. W., et al. 1994, ApJ, 422, 906

Solanki, S. K., del Toro Iniesta, J. C., Woch, J., et al. 2020, A\&A, 642, A11

Spice Consortium (Anderson, M., et al.) 2020, A\&A, 642, A14

Starck, J.-L., \& Murtagh, F. 1994, A\&A, 288, 342

Starck, J. L., \& Murtagh, F. 2002, Astronomical Image and Data Analysis (Springer-Verlag)

Teriaca, L., Banerjee, D., Falchi, A., Doyle, J. G., \& Madjarska, M. S. 2004, A\&A, 427, 1065

Thompson, W. T. 2006, A\&A, 449, 791

Tiwari, S. K., Panesar, N. K., Moore, R. L., et al. 2019, ApJ, 887, 56

Warren, H. P., \& Winebarger, A. R. 2000, ApJ, 535, L63 


\section{Appendix A: Data set description}

As part of the instrument's commissioning, the two EUI HRI telescopes were operated, for the first time, in parallel at a fast imaging cadence on 30 May 2020. The Solar Orbiter was located at $31.5^{\circ}$ west in solar longitude from the Earth-Sun line (see Fig. 1) and at a distance of $0.556 \mathrm{AU}$ from the Sun. From this vantage point, the HRI $\mathrm{EUV}_{\mathrm{EU}}$ angular pixel size of $0.492^{\prime \prime}$ corresponds to $198 \mathrm{~km}$ on the solar surface. The $\mathrm{HRI}_{\text {Lya }}$ angular pixel size of $0.514^{\prime \prime}$ corresponds to $207 \mathrm{~km}$. HRI $\mathrm{EUV}_{\mathrm{LV}}$ effectively achieves a two-pixel resolution of $0.984^{\prime \prime}$, while for $\mathrm{HRI}_{\mathrm{Lya}}$, diffraction from the $30 \mathrm{~mm}$ aperture and the type of detector limited the goal to $2^{\prime \prime}$, with an effective resolution estimated now of about $3^{\prime \prime}$. Both HRI cameras produce $2048 \times 2048$ pixel images that correspond to a $16.8 \times 16.8^{\prime} \mathrm{FOV}$.

The data analysed for this study were acquired between 14:54:00 UT and 14:58:20 UT and consist of two sequences of 50 images each, taken simultaneously by the two HRIs at $5 \mathrm{~s}$ cadence. The sequence of $\mathrm{HRI}_{\mathrm{EUV}}$ images started $15 \mathrm{~s}$ earlier. $\mathrm{HRI}_{\mathrm{EUV}}$ images were acquired with $3 \mathrm{~s}$ exposure while the $\mathrm{HRI}_{\text {Lya }}$ images were exposed for $1 \mathrm{~s}$. The data were acquired with lossless compression and processed to Level 2 FITS files as distributed in the SolO/EUI Data Release $1.0^{2}$.

We also used the data acquired by the AIA instrument (Lemen et al. 2012) on board the SDO (Pesnell et al. 2012) in Earth orbit. The AIA bandpass at $17.1 \mathrm{~nm}$ is similar but not identical to the HRI $\mathrm{EUV}_{\mathrm{EV}}$ and FSI bandpasses at $17.4 \mathrm{~nm}$. The detailed differences in bandpasses are not considered here but these will be the subject of future investigations (Gissot \& Auchère priv. comm.). The angular pixel sizes of AIA and HRIEUV are comparable, but given the difference in heliocentric distance, EUI provides a factor of 2 improvement in spatial resolution. The difference in heliocentric distance results also in difference in light travel time of $220 \mathrm{~s}$, meaning that EUI images acquired at time $T$ are to be compared with AIA images acquired at $T+220 \mathrm{~s}$. The AIA imaging cadence is $12 \mathrm{~s}$, hence, the closest in time AIA and EUI images can be up to $5 \mathrm{~s}$ off. We used despiked AIA images. In order to subtract residual thermal signal in faint regions, we subtracted for each quadrant the mean of a $100 \times 100$ pixels box in the corresponding corner.

To compare EUI and AIA across the whole FOV, we use images from both instruments that are remapped to Carrington coordinates. In this process, the HRI images were re-sampled with bi-cubic interpolation on a regular $2400 \times 2400$ grid with a 0.0163 heliographic degrees $(198 \mathrm{~km})$ pitch, thus preserving the HRI $_{\text {EUV }}$ resolution.

No extra data points are created in the conversion from the original $2048 \times 2048$ to the $2400 \times 2400$ frame as the extra pixels correspond to a dark border around the actual FOV (see top frame of Fig. 5). Following an optimisation process, the radius of the Carrington projection sphere was chosen at $1.004 R_{\odot}$, or $2.8 \mathrm{~mm}$ above the photosphere, in order to take out the average parallax over the field of view between the campfires seen in HRI $_{\text {EUV }}$ and AIA.

Individual events can still display a parallax between their HRI $_{\text {EUV }}$ and AIA images when their height deviates from this average. This can be an explanation for the mismatch seen in Fig. C.5 if this campfire is located exceptionally high above the photosphere.

\footnotetext{
2 https://doi.org/10.24414/wvj6-nm32
}

\section{Appendix B: Automated detection}

We use an automated detection method to separate these spacetime events from the random intensity fluctuations caused by the various sources of noise in the acquisition chain. In order to determine the statistical significance of a space-time blob of intensity, one must assume a model of the noise present in the data. In the following, we considered only the photon shot noise, which is expected to dominate the other sources of noise in HRI $\mathrm{EUV}_{\mathrm{EU}}$ (Rochus et al. 2020). While a detection in space-time directly would be desirable, in the present work we determined the statistical significance of small features spatially and tracked them in time through the sequence. The detection scheme applied to the stack of 50 Carrington projected images was as follows:

1. Small features are separated from the photon shot noise using the first two scales of a dyadic 'à trous' wavelet transform of the images using a $B_{3}$ spline scaling function (e.g., Starck \& Murtagh 2002). In Starck \& Murtagh (1994), the wavelet coefficients at each scale are considered significant when they are greater than $n$ times the root-meansquare amplitude expected from Gaussian white noise. In this study, we use the extension to Poisson statistics proposed by Murtagh et al. (1995), with $n=5$ sigma thresholding. The thresholding of the wavelet coefficients results in a binary cube.

2. The 6-connected voxels of the binary cube are clustered into numbered regions. Each region defines an event.

3. For each event, physically relevant parameters are computed, such as projected area, duration, and the parameters of the ellipse of the same second moments.

The above procedure with 2 wavelet scales and $n=5$ sigma thresholding, applied on the sequence of $50 \mathrm{HRI}_{\mathrm{EUV}}$ images resulted in 1467 campfires.

\section{Appendix C: Example campfires}

We compare the morphology of a few individual campfires as observed in $\mathrm{HRI}_{\mathrm{EUV}}, \mathrm{HRI}_{\mathrm{Lya}}$ and in the SDO/AIA bandpasses at $21.1 \mathrm{~nm}, 19.3 \mathrm{~nm}, 17.1 \mathrm{~nm}$, and $30.4 \mathrm{~nm}$. No clear event signatures were identified in any of the other SDO/AIA bandpasses.

The following figures show examples of individual campfires. In each figure, the top row corresponds to $\mathrm{HRI}_{\mathrm{EUV}}$ and $\mathrm{HRI}_{\text {Lya }}$ subfield images, while the panels on the second row show SDO/AIA subfield images, from left to right at respectively $21.1 \mathrm{~nm}, 19.3 \mathrm{~nm}, 17.1 \mathrm{~nm}$, and $30.4 \mathrm{~nm}$. Each panel corresponds to nearly $(10 \mathrm{~mm})^{2}$ on the Sun $(50 \times 50$ pixels, each with a footprint on the Sun of $(198 \mathrm{~km})^{2}$, except the two panels top left in each figure that show the $(60 \mathrm{~mm})^{2}$ neighbourhood in which the campfire takes place. The black rectangle shows the maximal extension in the $x$ and $y$ direction over which each campfire is detected over its duration.

As expected from the histograms in Fig. 2, most campfires are very close to the $\mathrm{HRI}_{\mathrm{EUV}}$ resolution limit. In this section we do not consider events detected as a single pixel or appearing in a single frame. Even then, most campfires appear as essentially dot-like of only a few pixels but their existence is beyond doubt as we can see their evolution over several images. In Figs. C.1, C.2, and 3, we show examples of such dot-like campfires. In each of the cases, there is no corresponding localised brightening in $\mathrm{HRI}_{\text {Lya }}$. A comparison with simultaneous AIA images shows that these smallest events can be identified in the displayed AIA wavelengths as well, although they appear weaker, fuzzier, and typically bigger, which is most likely an 
D. Berghmans et al.: Extreme-UV quiet Sun brightenings observed by the Solar Orbiter/EUI

effect of the lower spatial resolution. Without the indication from $\mathrm{HRI}_{\mathrm{EUV}}$, most of these events would remain unnoticed among noise fluctuations.

Amongst the slightly bigger campfires (e.g. Figs. C.3 and 4), we see loop-like events with an aspect ratio of 2 to 4 and a length of up to 10 pixels $(2 \mathrm{Mm})$. Again, there is no corresponding localised brightening in $\mathrm{HRI}_{\text {Lya }}$ but the events can be identified in simultaneous AIA images, though they appear weaker and fuzzier, thereby often hiding their elongated structure.
For the biggest campfires, $\mathrm{HRI}_{\mathrm{EUV}}$ often shows complex internal structure, which is missing from the corresponding AIA images due to the difference in resolution (Fig. C.4). This internal structure can possibly be interpreted as a group of nearby loops brightening up, either because of some local source in their neighbourhood, or because of mutual interactions. A suggestive example of the latter is the event shown in Fig. C.5. In the evolution of this event (Fig. C.6), we can see that the source location of the event (the black rectangle) later becomes the forking point of what could be interpreted as two interacting loops.

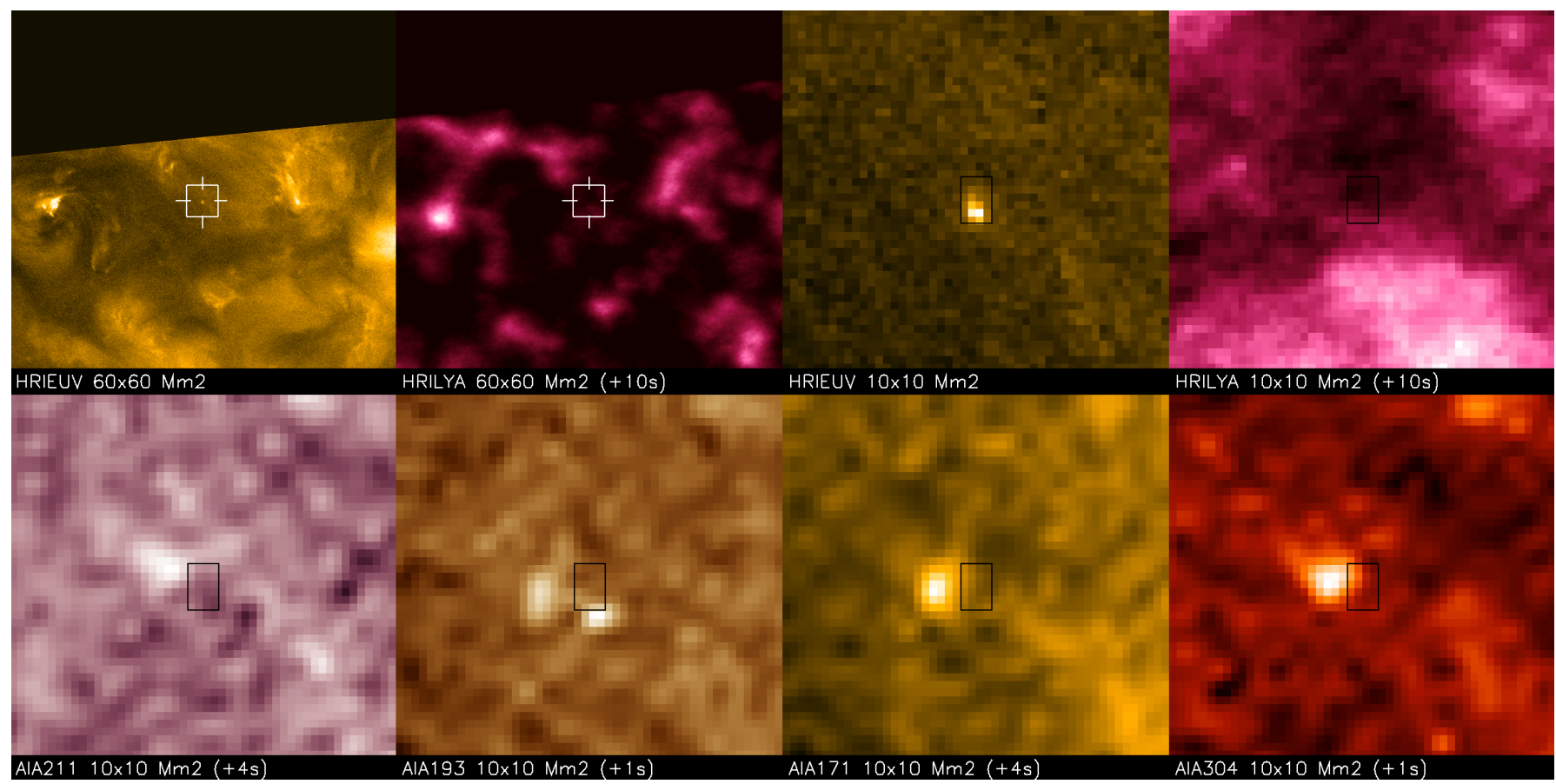

Fig. C.1. Example of a dot-like event. The white square in the two top-left panels corresponds to the field of view of the other, zoomed-in panels.

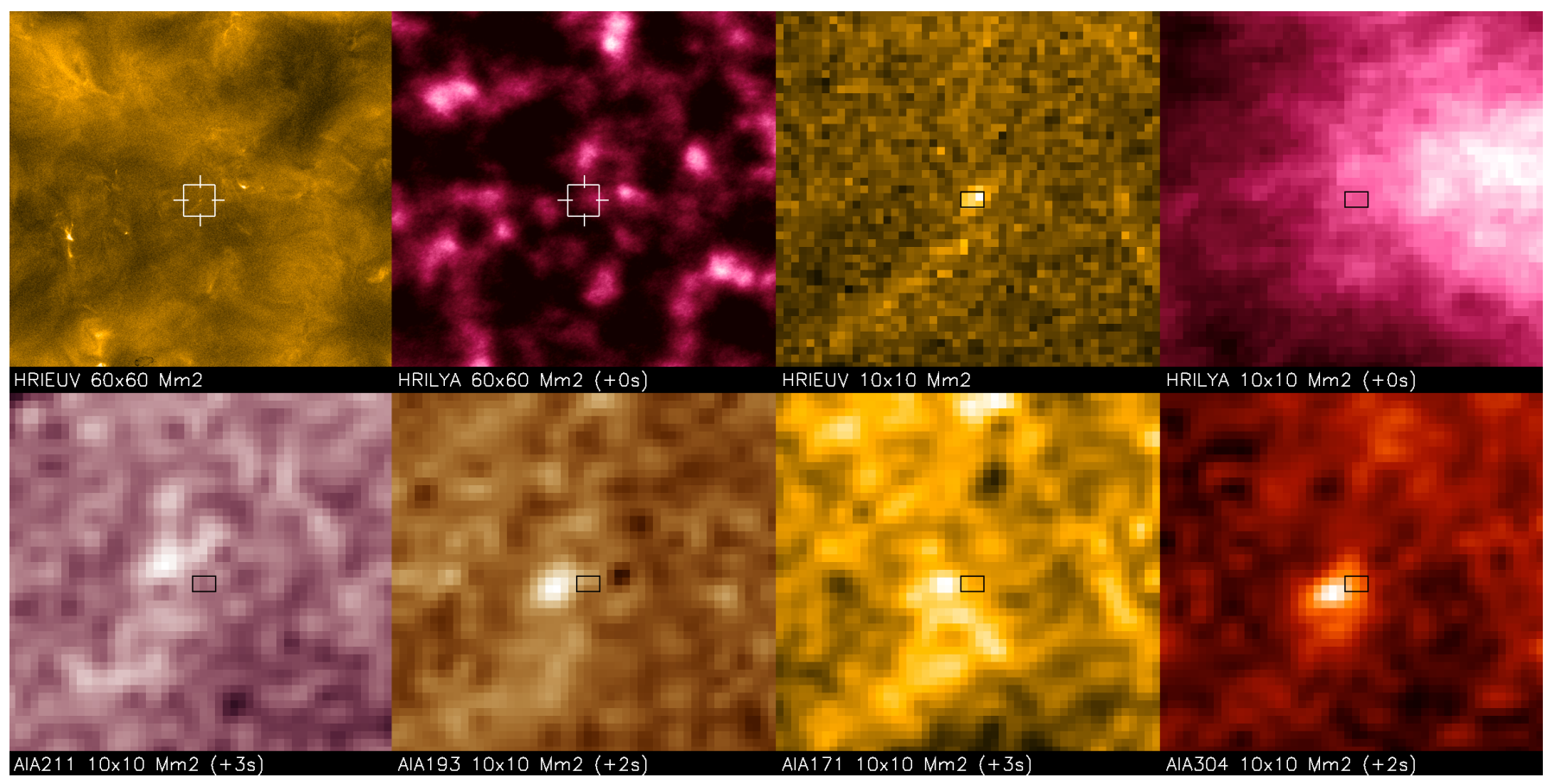

Fig. C.2. Example of a dot-like event. The white square in the two top-left panels corresponds to the field of view of the other, zoomed-in panels. 


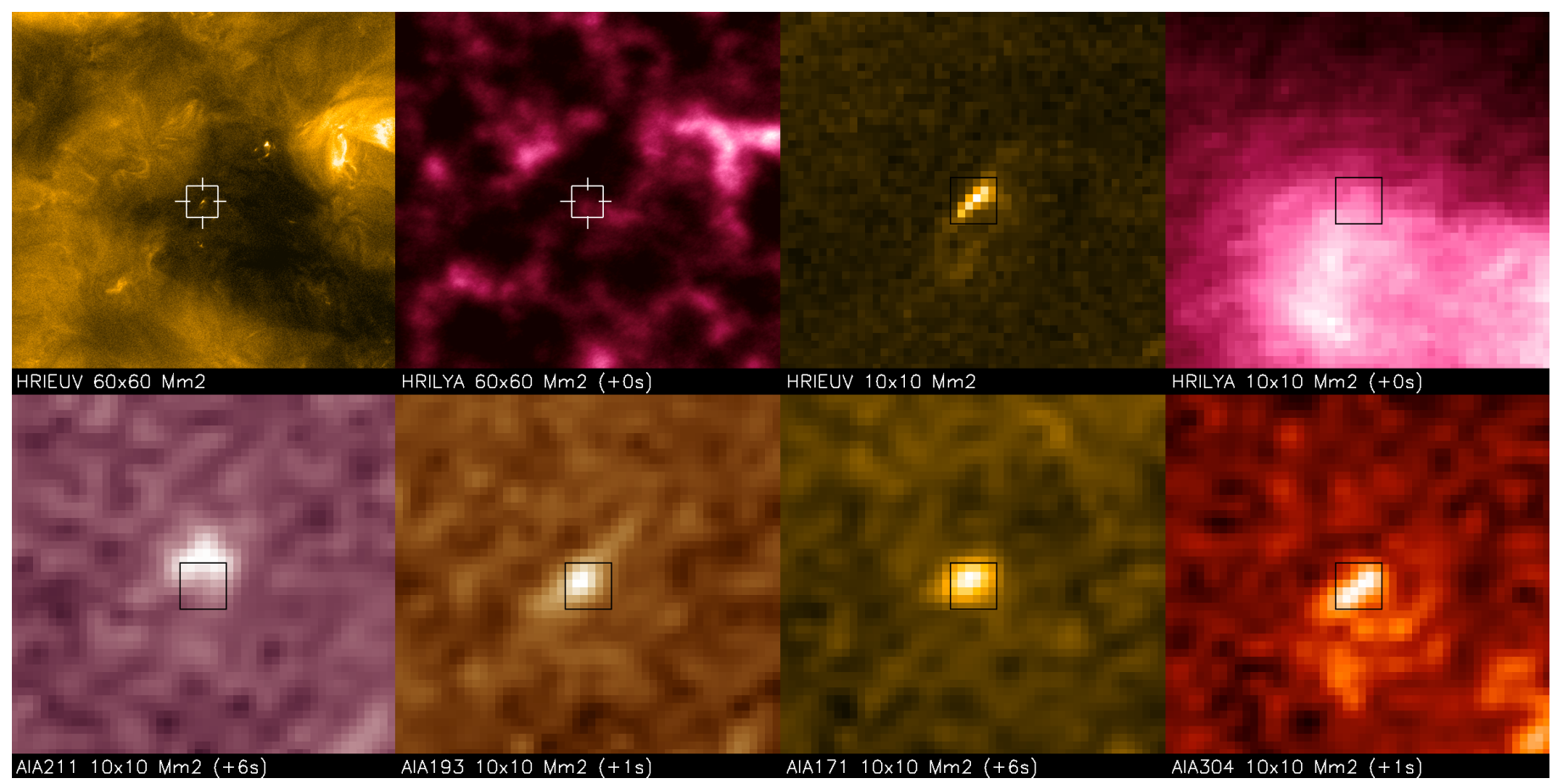

Fig. C.3. Example of a loop-like event. The white square in the two top-left panels corresponds to the field of view of the other, zoomed-in panels.

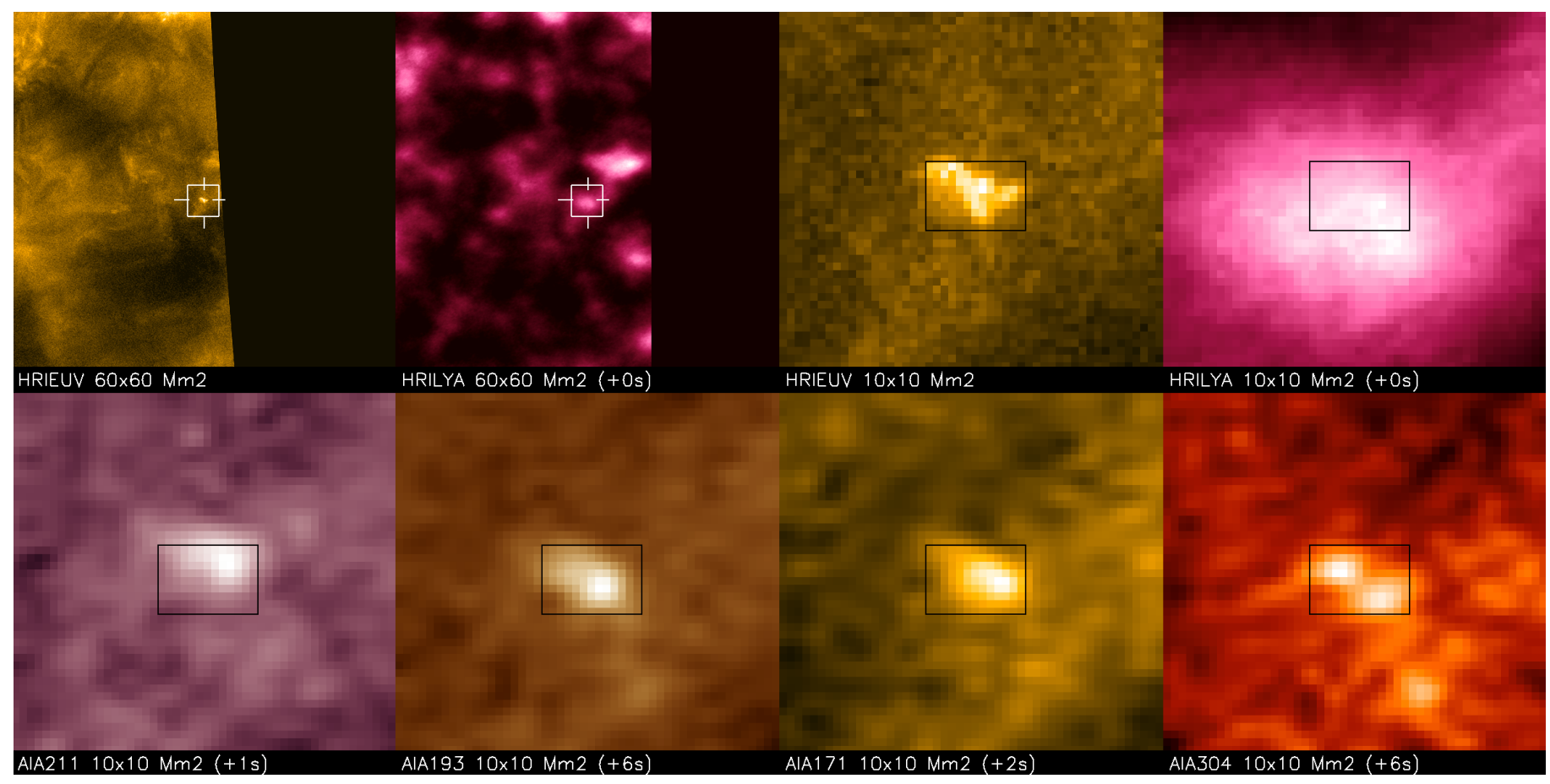

Fig. C.4. Example of complex event. The white square in the two top-left panels corresponds to the field of view of the other, zoomed-in panels. 
D. Berghmans et al.: Extreme-UV quiet Sun brightenings observed by the Solar Orbiter/EUI

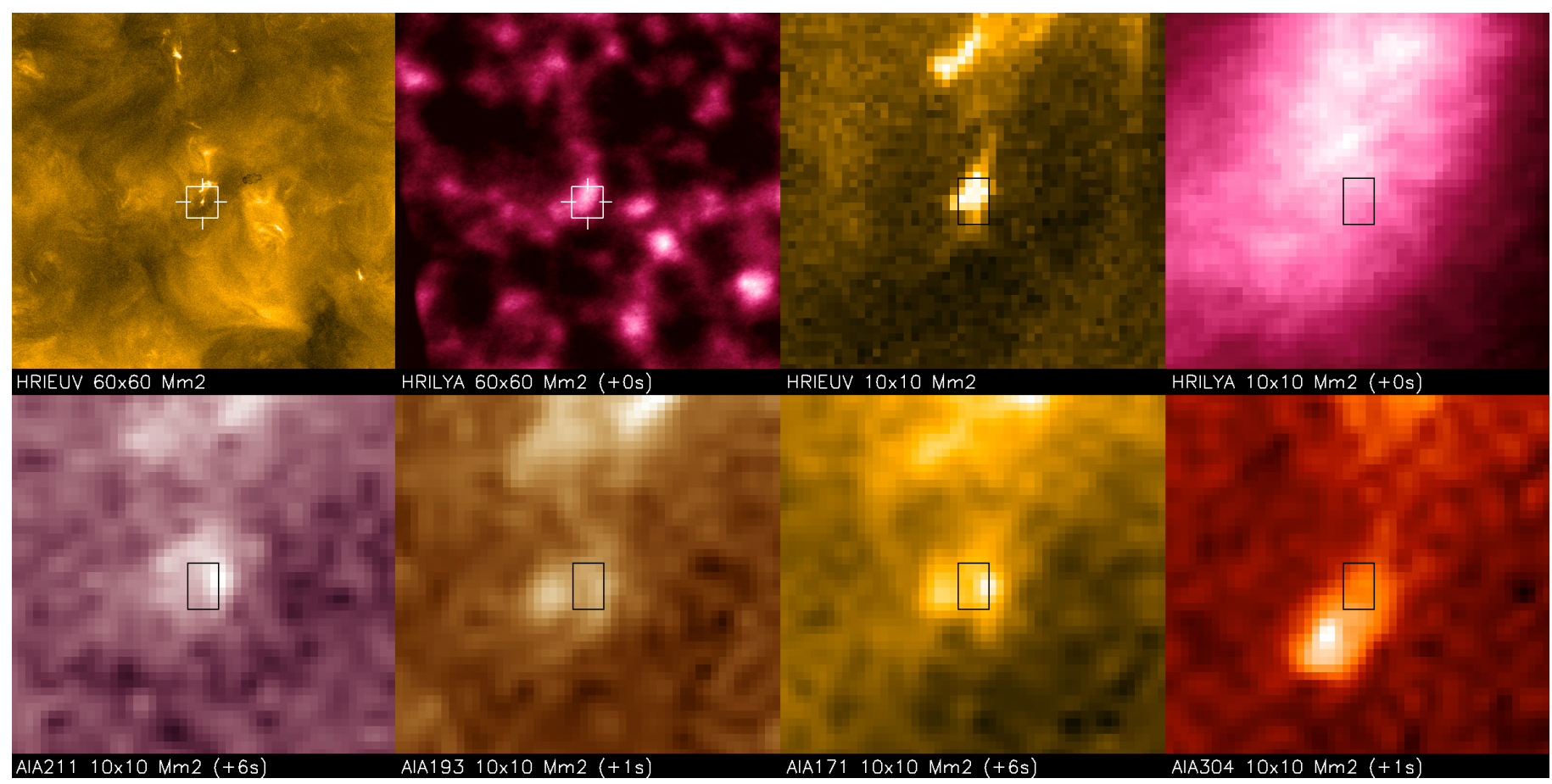

Fig. C.5. Example of complex event. The white square in the two top-left panels corresponds to the field of view of the other, zoomed-in panels.

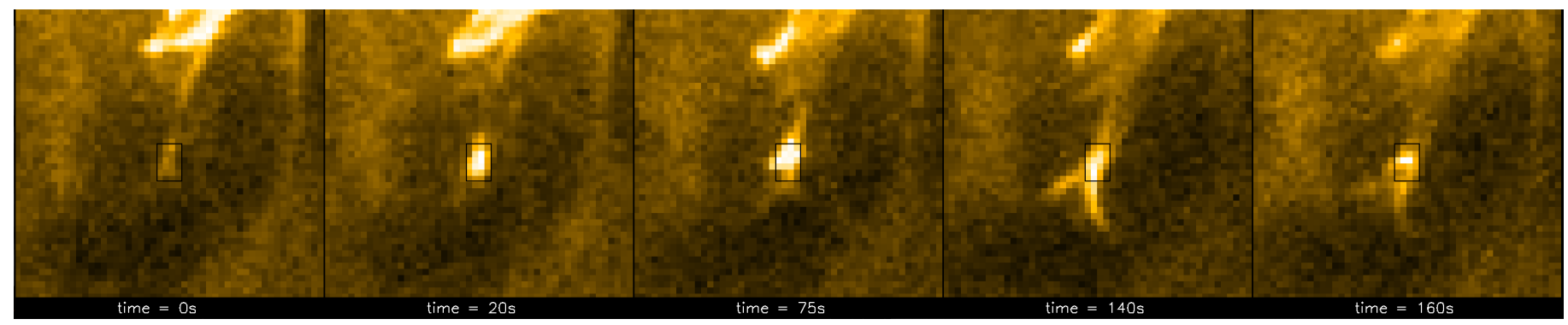

Fig. C.6. Time evolution of the event shown in Fig. C.5. The latter corresponds to $t=75 \mathrm{~s}$. Each frame corresponds to an area of $10 \times 10 \mathrm{~mm}$ and each pixel corresponds to an area of $197 \mathrm{~km} \times 197 \mathrm{~km}$.

\section{Appendix D: Height of campfires}

In order to determine the height of campfires above the photospheric surface, we performed triangulation on sixteen small-size campfires that could be identified on both $\mathrm{HRI}_{\mathrm{EUV}}$ (14:54:10 UT) $17.4 \mathrm{~nm}$ and AIA $17.1 \mathrm{~nm}$ (14:57:57 UT) images. The difference in time of the two images is chosen to accommodate for the light travel time to the two instruments, so that they image the same solar scene nearly simultaneously (to within $2 \mathrm{~s}$ ).

The method consists of identifying the same feature (pixel) in the two images (tie-pointing) and calculating the lines of sight that pass through the corresponding pixels in the two images. This allows calculating the position of the intersection point in 3D space (e.g. Inhester 2006). The triangulation was performed using the scc_measure.pro SolarSoft routine, and the location of the campfires in Stonyhurst coordinates (longitude, latitude, and height, $h$, from the Sun's centre; see Thompson 2006) was obtained. The half-width triangulation error $\delta h$ is related to the linear pixel sizes $\delta s_{1}$ and $\delta s_{2}$ of HRI $\mathrm{EUV}_{\mathrm{Ev}}$ and AIA, respectively, and can be calculated as follows:

$$
\delta h=\frac{\sqrt{\delta s_{1}^{2}+\delta s_{2}^{2}+2 \delta s_{1} \delta s_{2} \cos \gamma}}{2 \sin \gamma},
$$

where $\gamma$ is the separation angle between Solar Orbiter and SDO. On 30 May 2020, $\gamma=31.5^{\circ}$, one AIA pixel is $441 \mathrm{~km}$ and one $\mathrm{HRI}_{\mathrm{EUV}}$ pixel is $198 \mathrm{~km}$. The error is then $\delta h=600 \mathrm{~km}$ if one can localise a campfire to within one pixel in both $\mathrm{HRI}_{\mathrm{EUV}}$ and AIA images. This is sometimes difficult to do, so that the error may be up to four HRI EUV and two AIA pixels, which gives an error of $1500 \mathrm{~km}$. This is the random error linked to the limited resolution of the telescopes.

On top of the random error for the height, there is a systematic error due to an uncertainty of a few arcsec on the pointing of the $\mathrm{HRI}_{\mathrm{EUV}}$ telescope with respect to the centre of the Sun. We estimated that this uncertainty would lead to a systematic error of $\pm 900 \mathrm{~km}$ on the derived heights. 